\title{
The interaction between a particle and an advancing solidification front
}

\author{
A.W. Rempel*, M.G. Worster \\ Department of Applied Mathematics and Theoretical Physics, Institute of Theoretical Geophysics, University of Cambridge, Silver Street, \\ Cambridge CB3 9EW, UK
}

Received 17 March 1999; accepted 26 April 1999

Communicated by A.A. Chernov

\begin{abstract}
We derive analytical expressions for the velocity of an insoluble particle near an advancing solidification front when the intermolecular interactions are described by a power-law dependence between the film thickness and the undercooling. We predict that the maximum particle velocity, which corresponds to the lowest solidification velocity at which particle trapping occurs, depends inversely on the particle radius. The critical velocity is less sensitive to the temperature gradient and the precise dependence changes with different interaction types. When the critical velocity is exceeded, the particle becomes trapped within the solid region after being pushed slightly ahead of its initial position. The predicted particle displacement is typically only a fraction of the particle radius. Particle buoyancy can enhance or reduce the tendency for the particle to be captured, though it does not affect the parametric dependence of the critical velocity on the particle radius and the temperature gradient. (C) 1999 Elsevier Science B.V. All rights reserved.
\end{abstract}

PACS: 64.70.Dv; 68.15. + e; 68.45.Gd; 81.30.Fb

Keywords: Insoluble particle; Solidification front; Particle trapping; Premelting; Solid-liquid interface; Engulfment

\section{Introduction}

When a solidification front advances towards a foreign particle which is suspended in the melt, two different types of behaviour can result. In some cases the solid-liquid interface deforms around the particle, thereby trapping it within the growing solid region. If the solidification rate is slow and the

\footnotetext{
* Corresponding author. Tel.: + 44-1223-339208; fax: + 441223-337918.

E-mail address: a.w.rempel@damtp.cam.ac.uk (A.W. Rempel)
}

particle is sufficiently small, however, the particle is pushed ahead of the interface so that it remains nearly surrounded by the bulk liquid. The eventual outcome of interactions between particles and solidification fronts have important implications for the effective bulk properties of the newly formed solid and the final distribution of particles. Hence the prediction of particle-interface behaviour is of interest to those who study the fabrication of composites reinforced with ceramic particles [1], the casting of alloys containing insoluble particles, the cryogenic preservation of biological materials [2], and the formation of ice lenses in frozen soils [3]. 
The dependence of the solidification-front behaviour on the particle size and solidification velocity was first reported by Corte [3]. Corte's interest in the frost-heaving characteristics of soils motivated him to perform a series of experiments in which he investigated the interaction of the ice-water interface with particles made of a variety of different geological materials. Uhlmann and colleagues [4] subsequently offered a theory that attempted to predict the type of behaviour that prevails at a given solidification velocity depending on material properties and the size of the particle. In their formulation they assumed that the chemical potential in the liquid near the particle is altered by the presence of the particle. The particle is repelled from the planar ice-liquid interface as long as the ice-particle separation is greater than a critical threshold which they took to be equal to a molecular diameter. When the solidification velocity is too rapid or the particle is too large, the separation decreases below this threshold and the particle becomes trapped in the growing solid. In a refinement of these ideas, Bolling and Cissé [5] noted that the viscous drag accompanying fluid motion around the particle is affected by the deformation of the ice-liquid interface as the local melting temperature is lowered by the presence of the particle.

Gilpin [6] abandoned the assumption that the particle is only trapped once it advances to within a molecular diameter of the interface. He had earlier developed a theory to describe the behaviour of the liquid-like layer that forms to separate ice from a foreign substrate because of the influence of the substrate on the chemical potential, and the effects of interface curvature [7]. Using this theory he derived an equation for the variation of liquidfilm thickness with angular distance from the centre of the particle. After solving for the interface shape, he balanced the effective particle-rejection pressure with the opposing effects of the particle weight and viscous drag in the fluid. This enabled him to calculate the maximum solidification velocity that allows the particle to remain in front of the interface as solid continues to form. Unfortunately, the calculation of the interface shape using Gilpin's formulation is complicated and it is difficult to compare his predictions with experimental data. In addition, Gilpin's treatment of the liquid-like layer relies on contentious assumptions about the relationship between the chemical potential in the liquid and the distance from the particle surface.

In the Soviet Union meanwhile, Chernov and his colleagues $[8,9]$ recognised that intermolecular forces acting between the particle, the solid, and the liquid must be responsible for particle rejection. In the simplest case the dominant forces are due to van der Waals interactions (e.g. Ref. [10]). The intermolecular forces results in what has previously been called a disjoining pressure that can act to repel the particle from the interface, while viscous forces associated with liquid flow around the particle tend to promote particle encapsulation within the growing solid. The region near the base of the particle is responsible for the largest contributions to the forces on the particle. In their first model, Chernov and his colleagues treated the forces as being locally dominated so that the predicted geometry of the deformed solid-liquid interface takes the form of a paraboloid. This enabled them to derive approximate analytical expressions for the particle motion as a function of its size and the temperature gradient. As a refinement to this initial model, they also presented a second formulation that treated the interface geometry as the intersection of a paraboloid with a plane.

In the current work, we revisit this problem to predict the behaviour of a particle near a solid-liquid interface. We focus on the essence of the problem and avoid the complications that could be introduced by secondary effects such as differing thermal conductivities between the system components. Much of the formulation follows the spirit of that proposed by Chernov and colleagues $[8,9]$ and recently reviewed by Worster and Wettlaufer [11]. We deviate from the previous analysis in that we avoid the need for ad hoc approximations to the interface geometry. We begin by examining the force balance on a single particle for the case where the intermolecular forces are dominated by nonretarded van der Waals interactions and the particle is large enough that the effects of interface curvature can be neglected. This leads to expressions for the particle velocity as a function of the film thickness or particle position. Simplified expressions for the particle velocity that are valid at large and small film thickness are also presented. 
We determine the critical solidification velocity that must be exceeded in order for a particle of a given size to be captured. When this solidification velocity is surpassed, the question becomes one of how far the particle will travel before it is finally trapped within the solid. Next, we investigate how the predictions are changed if the intermolecular forces are dominated by different types of power law interactions. Finally, we discuss briefly how the effects of particle buoyancy can modify the velocity of larger particles. The goal throughout is to illuminate the basic physical processes that control the behaviour of the system in the simplest possible circumstances.

\section{The forces on a particle}

Consider an ice front advancing at a constant solidification velocity $V$ towards a spherical particle of radius $R$ subjected to a uniform temperature gradient $G$ as depicted in Fig. 1. Initially, the particle is at rest with its centre a distance $H$ above the melting temperature isotherm $T_{\mathrm{m}}$. As $H$ decreases and $H-R$ becomes comparable to the distance where intermolecular forces are important, interac-

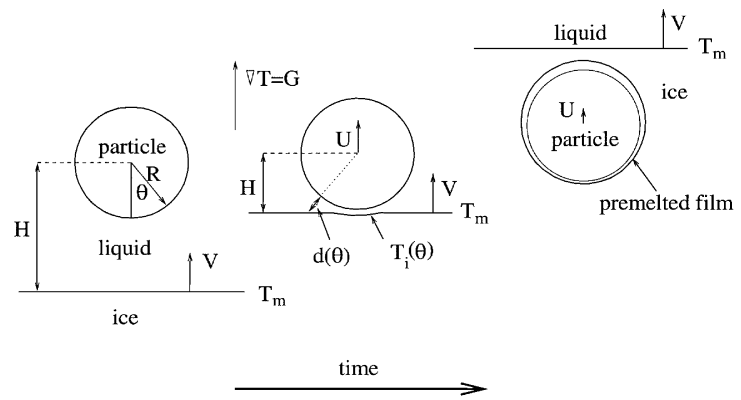

Fig. 1. Schematic diagram of a freezing front impinging upon a foreign particle in a temperature gradient $G$. Initially the separation $H$ is large enough that the solid-liquid interface is unaffected by the presence of the particle. As the front nears the particle, the interface deforms to allow a premelted film of thickness $d(\theta)$ to form and separate the particle from the phase boundary. The interface temperature $T_{\mathrm{i}}(\theta)$ is depressed below the bulk melting temperature $T_{\mathrm{m}}$ and the particle is pushed ahead of the interface at velocity $U$. If the solidification velocity $V$ exceeds some critical value $V_{\mathrm{c}}$ the particle is eventually trapped within the ice. tions with the particle cause the ice-liquid interface to deform and the particle begins to move. The rate of particle motion $U$ is determined by a balance between the thermomolecular pressure that separates the particle from the ice surface, and the lubrication force associated with fluid flow in the intervening liquid film. Both of these forces increase in strength as the film thickness is reduced and the ice-liquid interface becomes increasingly deformed. The particle accelerates until its velocity either matches the solidification velocity or it reaches the critical velocity $V_{\mathrm{c}}$ which corresponds to the minimum solidification velocity required for particle trapping. If the solidification velocity $V$ is less than $V_{\mathrm{c}}$ the particle reaches an equilibrium separation from the interface where its velocity remains constant at $U=V$. In the case where $V$ is greater than $V_{\mathrm{c}}$, however, $H$ continues to decrease and the film thickness is further reduced; this causes the supply of liquid to be increasingly impeded by viscous effects so that the particle velocity decreases and eventually the particle is completely surrounded by ice.

The deformation of the ice-liquid interface and the presence of the liquid film which separates the particle from the ice surface are both manifestations of the phenomenon known as interfacial premelting (e.g. Ref. [12,13]). When the particle is large enough that the effects of interface curvature can be neglected, the thickness of the film is related to the temperature of the interface $T_{\mathrm{i}}$ according to

$d=\lambda\left(\frac{T_{\mathrm{m}}}{T_{\mathrm{m}}-T_{\mathrm{i}}}\right)^{1 / v}$,

where $T_{\mathrm{m}}$ is the bulk melting temperature ( $273.15 \mathrm{~K}$ for ice) and $\lambda$ is a lengthscale proportional to the interaction strength. The exponent $1 / v$ depends on the origin of the dominant interfacial interactions. Initially we assume that nonretarded van der Waals forces control $d$, in which case $v=3$. Later we generalise the analysis to consider other types of interactions which can be modelled using different values for $v$. When $d$ is very small, entropic effects can produce film thickness variations with a different functional form to that given by Eqs. (1)[9]. The presence of dissolved impurities can also alter the relationship between the film thickness 
and the interface temperature $[9,14]$. For the present analysis we shall avoid these complications and confine our attention to systems where the film thickness is described by a power law of the form given by Eq. (1).

When the thermal conductivity of the particle is different from that of the melt, the temperature field can become distorted near the particle. This causes the bulk melting temperature isotherm to curve outwards towards the particle if the particle is more insulating than the melt, while a particle made of a good thermal conductor causes the $T_{\mathrm{m}}$ isotherm to deform in the opposite sense. Experimental evidence suggests that such thermal effects can influence the critical velocity $V_{\mathrm{c}}$ by promoting the capture of high-conductivity particles and reducing the tendency of insulating particles to be trapped within the melt [15]. We defer analysis of this complicated thermal problem to future research, and treat the thermal conductivities of the particle, the melt, and the solid as equal while the temperature gradient is assumed constant. This allows us to write the temperature at the solid-liquid interface as

$$
T_{\mathrm{i}}(\theta, t)=T_{0}-G(R+d) \cos \theta-G \int_{0}^{t}(V-U(\tau)) \mathrm{d} \tau
$$

where $T_{0}$ is the initial temperature at the centre of the particle. Combining Eqs. (1) and (2) with $v=3$ leads to

$$
\begin{aligned}
\frac{l^{4}}{d^{3}} & =(R+d) \cos \theta-\left(\frac{T_{0}-T_{m}}{G}-\int_{0}^{t}(V-U(\tau)) \mathrm{d} \tau\right) \\
& =(R+d) \cos \theta-H
\end{aligned}
$$

where $H(t)$ is the height of the particle centre above the $T_{\mathrm{m}}$ isotherm, and $l \equiv\left(\lambda^{3} T_{\mathrm{m}} / G\right)^{1 / 4}$ is a lengthscale that is characteristic of the film thickness. When the centre of the particle is one radius distant from the $T_{\mathrm{m}}$ isotherm (i.e. $H=R$ ) the film thickness immediately beneath the particle at $\theta=0$ is $d_{0}=l$.

Above the deformed region of the ice-liquid interface the film thickness is very much less than the particle radius and the thermomolecular pressure can be written as

$P_{\mathrm{T}}=\frac{A}{6 \pi d^{3}}=\frac{\rho_{\mathrm{s}} q_{\mathrm{m}} \lambda^{3}}{d^{3}}$

where $A$ is the effective Hamaker constant for interactions between the particle and the ice with an intervening liquid film, and $\rho_{\mathrm{s}}$ and $q_{\mathrm{m}}$ are the ice density and latent heat of ice, respectively. The Hamaker constant can either be calculated from spectral data for the dielectric functions of water, ice and the particle material of interest $[16,17]$, or it can be deduced from experimental measurements [13]. Eq. (4) indicates that the thermomolecular pressure attains its maximum immediately beneath the particle where the premelted film is thinnest and the particle surface is nearly parallel to the interface. Integrating $P_{\mathrm{T}}$ over the interfacial surface gives the vertical component of the thermomolecular force as

$$
\begin{aligned}
F_{\mathrm{T}} & =2 \pi R^{2} \int_{0}^{\theta_{\mathrm{c}}} \sin \theta \cos \theta P_{\mathrm{T}} \mathrm{d} \theta \\
& =\left(2 \pi R^{2}\right) \rho q_{\mathrm{m}} \lambda^{3} \int_{0}^{\theta_{\mathrm{c}}} \frac{\sin \theta \cos \theta}{d^{3}} \mathrm{~d} \theta .
\end{aligned}
$$

The largest contributions to the thermomolecular force come from the region near the base of the particle where $\theta$ is small. The integral in Eq. (5) is relatively insensitive to the precise choice of the upper limit of integration $\theta_{c}$ provided that the film thickness at $\theta_{\mathrm{c}}$ is much greater than the film thickness immediately beneath the particle $d_{0}$.

Since the particle is in motion, mass conservation dictates that liquid must flow in the thin film that separates the particle from the ice front. Near the base of the particle the film thickness is much less than the particle radius; thus a lubrication approximation can be used to find the volume flux as

$q=-\frac{\pi d^{3} \sin \theta}{6 \mu} \frac{\mathrm{d} P_{1}}{\mathrm{~d} \theta}$

where $\mu$ is the dynamic viscosity and $P_{1}$ is the pressure in the liquid film. If we neglect the density difference between liquid water and ice, mass conservation implies that the volume of liquid transported past the angular position $\theta$ in a time $\mathrm{d} t$ is equal to the volume swept out beneath the particle 
as it travels the corresponding distance $\mathrm{d} z$

$q \mathrm{~d} t=-\pi(R \sin \theta)^{2} \mathrm{~d} z$

Together with Eq. (6) this allows the liquid pressure to be written as

$P_{1}(\theta)=\int_{\theta_{c}}^{\theta} \frac{6 \mu R^{2} \sin \phi U}{d^{3}} \mathrm{~d} \phi$,

where $U \equiv \mathrm{d} z / \mathrm{d} t$ is the particle velocity and the integration limits are chosen so that $P_{1}\left(\theta_{c}\right)$ is set to zero. The vertical component of the lubrication force is found by integrating the liquid pressure over the deformed surface of the ice-liquid interface to give

$F_{\mu}=\left(2 \pi R^{2}\right) 6 \mu R^{2} U \int_{0}^{\theta_{\mathrm{c}}} \sin \theta \cos \theta\left(\int_{\theta_{\mathrm{c}}}^{\theta} \frac{\sin \phi}{d^{3}} \mathrm{~d} \phi\right) \mathrm{d} \theta$.

\section{Particle velocity}

To simplify the analysis, the density of the particle is set equal to that of the melt so that the buoyancy force is zero. We will demonstrate later that this is a trivial constraint which can be removed with negligible effect for many problems of interest. In the absence of any additional forces the sum of $F_{\mathrm{T}}$ and $F_{\mu}$ must be zero. From Eqs. (5) and (9) then, the steady-state particle velocity must satisfy

$$
U=-\frac{\rho q_{\mathrm{m}} \lambda^{3}}{6 \mu R^{2}} \frac{\int_{0}^{\theta_{\mathrm{c}}} \sin \theta \cos \theta d^{-3} d \theta}{\int_{0}^{\theta_{\mathrm{c}}} \sin \theta \cos \theta\left(\int_{\theta_{\mathrm{c}}}^{\theta} \sin \phi d^{-3} \mathrm{~d} \phi\right) \mathrm{d} \theta},
$$

where the film thickness can be determined from Eq. (3). It is convenient to nondimensionalise the particle height by its radius so that $H=h R$, while the film thickness is scaled with $l$ so that $d=\delta l$. This enables Eq. (3) to be written as

$$
\frac{\varepsilon}{\delta^{3}}=(1+\varepsilon \delta) \cos \theta-h,
$$

where $\varepsilon$ is a small parameter equal to the ratio of the film thickness length scale to the particle radius (i.e. $\varepsilon \equiv l / R$ ). To choose a velocity scale we recall the definition for $l$ and let

$$
W \equiv \frac{\rho q_{\mathrm{m}} l^{3} G}{6 \mu T_{\mathrm{m}} R}=\frac{\rho q_{\mathrm{m}} \lambda^{9 / 4} G^{1 / 4}}{6 \mu T_{\mathrm{m}}^{1 / 4} R}
$$

which is inversely proportional to the particle radius, but only weakly dependent on the temperature gradient. We shall see that, to leading order, the critical velocity is simply a multiple of this velocity scale. Hence the critical velocity is inversely proportional to the particle radius and dependent on the one fourth power of the temperature gradient. From Eq. (10) the dimensionless particle velocity becomes

$$
u \equiv \frac{U}{W}=-\frac{\varepsilon \int_{0}^{\theta_{\mathrm{c}}} \sin \theta \cos \theta \delta^{-3} d \theta}{\int_{0}^{\theta_{\mathrm{c}}} \sin \theta \cos \theta\left(\int_{\theta_{\mathrm{c}}}^{\theta} \sin \phi \delta^{-3} \mathrm{~d} \phi\right) \mathrm{d} \theta} .
$$

Eqs. (11) and (13) can be solved together to determine the particle velocity either as a function of the dimensionless film thickness immediately beneath the particle $\delta_{0}$, or as a function of the dimensionless particle height $h$. The integrals in Eq. (13) are easily evaluated once they are transformed so that the integration takes place over the dimensionless film thickness rather than the angular position. Finding $\mathrm{d} \delta / \mathrm{d} \theta$ from an implicit differentiation of Eq. (11) and substituting for $\cos \theta$ enables us to integrate Eq. (13) and find that

$$
u \approx\left(\frac{1}{2 \delta_{0}^{6}}+\frac{1}{2 \delta_{0}^{2}}\right)\left(\frac{1}{6 \delta_{0}^{9}}+\frac{2}{5 \delta_{0}^{5}}+\frac{1}{2 \delta_{0}}\right)^{-1}
$$

where $\delta_{0}$ represents the dimensionless film thickness at $\theta=0$. Eq. (14) is accurate to leading order in $\varepsilon$, which is consistent with the other approximations inherent in the present formulation.

In Fig. 2 the solid line shows the dimensionless particle velocity $u$ from Eq. (14) as a function of $\delta_{0}$. At large film thickness the interaction between the particle and the interface is weak and the particle motion is slow. The particle velocity increases as the film thickness decreases until a maximum of 


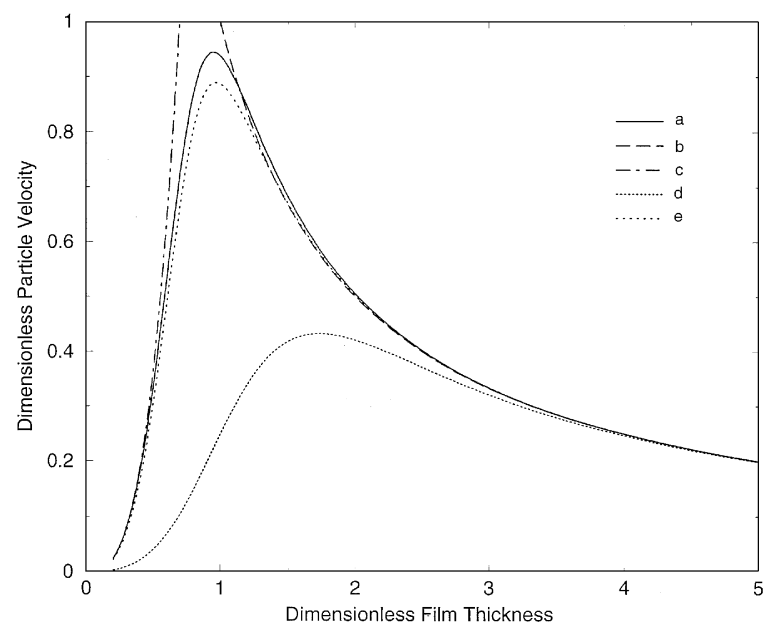

Fig. 2. Dimensionless particle velocity $u$ as a function of the dimensionless film thickness $\delta_{0} \equiv d_{0} / l$. The solid line (a) shows the leading order behaviour that is predicted from Eq. (14). The dashed line (b) on the right denotes the approximate velocity that is calculated by assuming that the phase boundary remains planar. The dot-dashed line (c) on the left gives the approximate velocity when the temperature on the particle surface is substituted for that along the phase boundary. The lower dotted line (d) shows the predictions of Chernov and colleagues [8] for the particle velocity when the phase boundary is modelled as a paraboloid. The upper dotted line (e) displays the predictions of Chernov and colleagues [8] for the case where the interface has the form of a paraboloid intersecting with a plane.

$v_{\mathrm{c}} \approx 1$ is reached in the vicinity of $\delta_{0}=1$ where the base of the particle is level with the bulk meltingtemperature isotherm (i.e. $H=R$ ). Recalling the definition of the velocity scale $W$ in Eq. (12), we see that when nonretarded van der Waals interactions dominate and $v=3$, the critical solidification velocity is proportional to $\lambda^{9 / 4} G^{1 / 4} R^{-1}$. Larger particles are captured at lower solidification velocities while higher temperature gradients tend to promote particle repulsion. Higher values of the interaction-strength length scale $\lambda$, which is proportional to the Hamaker constant, correspond to larger film thicknesses at a given temperature; fluid flow is less restricted and hence the critical solidification velocity is higher. To the left-hand side of this maximum for $u$, as the film thickness is further reduced the particle becomes increasingly surrounded by ice and viscous effects cause the particle velocity to decrease rapidly. To provide further insight into the behaviour of the system it is instructive to examine briefly two other simple approximations for $u$ that are valid for large and small values of $\delta_{0}$, respectively.

The presence of the particle first begins to affect the interface when $\delta_{0}$ is still much greater than unity, but much less than $\varepsilon^{-1}$. The interface deformation is minimal in this limit and the film thickness can be approximated using the distance from the particle to the undeformed plane of the $T_{\mathrm{m}}$ isotherm so that Eq. (11) reduces to

$\delta \approx \frac{h-\cos \theta}{\varepsilon \cos \theta}$

This expression for $\delta$ can be substituted into Eq. (13) and the approximate dimensionless particle velocity calculated with $\theta_{\mathrm{c}}=\pi / 2$. In this limit the dimensionless particle height is roughly $h \approx$ $1+\varepsilon \delta_{0}$ so that the leading order behaviour is given by $u \approx 1 / \delta_{0}$. The dashed line on the right-hand side of Fig. 2 shows that this approximate solution for $u$ compares well with that given by Eq. (14) when $\delta_{0}$ is greater than about two. However, by calculating the particle velocity without accounting for the deformation of the interface it is not possible to establish the critical solidification velocity at which the particle is captured. Previous authors (e.g. Refs. $[4,18]$ ) have avoided this problem by arbitrarily defining a cut-off distance that corresponds to an assumed minimum film thickness and stating that the velocity at that point is the critical solidification velocity. Typically it is assumed that the particle approaches the phase boundary to within a molecular diameter (i.e. about $10^{-4} \mu \mathrm{m}$ ). There is no theoretical justification for such an assumption. In fact, the current analysis indicates that the film thickness at the maximum particle velocity is roughly $d \approx l$ which depends on both the temperature gradient $G$ and the interaction-strength lengthscale $\lambda$, and is generally much greater than the molecular diameter of water. Under typical experimental conditions $\lambda \approx 10^{-4} \mu \mathrm{m}, G \approx 10^{4} \mathrm{~K} \mathrm{~m}^{-1}$, and $T_{\mathrm{m}} \approx 10^{2} \mathrm{~K}$ so that $l \approx 10^{-2} \mu \mathrm{m}$; for a particle with a radius of about $10 \mu \mathrm{m}$ we predict a critical velocity of $v_{\mathrm{c}} \approx 1 \mu \mathrm{s}^{-1}$ which is roughly consistent with experimental observations (see Fig. 3). If the particle were assumed to approach the interface to 


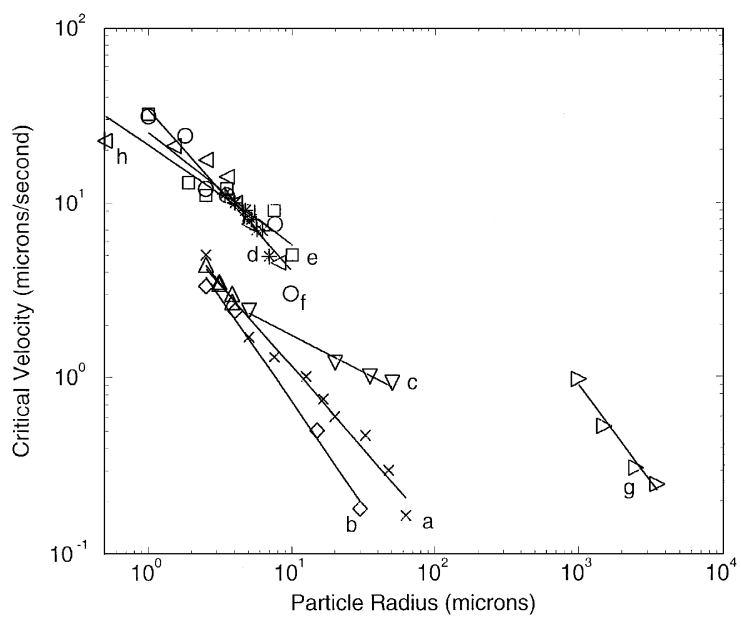

Fig. 3. The dependence of the critical velocity on the particle radius from a number of experimental studies. A best-fit line is shown running through each of the data sets. The different symbols correspond to the following particle materials in water: (a) copper with $G \approx 10^{4} \mathrm{~K} \mathrm{~m}^{-1}$ [19], the best-fit line has a slope of -0.9 ; (b) copper with $G \approx 10^{3} \mathrm{~K} \mathrm{~m}^{-1}$ [19], slope - 1.2; (c) tungsten with $G \approx 10^{4} \mathrm{~K} \mathrm{~m}^{-1}$ [19], slope - 0.4; (d) latex with $\mathrm{G} \approx 10^{4} \mathrm{Km}^{-1}$ [20], slope -1.0 ; (e) latex with $G \approx 4 \times$ $10^{4} \mathrm{~K} \mathrm{~m}^{-1}$ [21], slope -0.6 ; (f) latex with $G \approx 1.8 \times$ $10^{4} \mathrm{~K} \mathrm{~m}^{-1}$ [22], slope -0.9 ; $(\mathrm{g})$ nylon with $G \approx 200 \mathrm{~K} \mathrm{~m}^{-1}$ [23], slope -1.1 . The upward pointing triangles correspond to polystyrene particles in a succinonitrile melt with $G \approx 10^{4} \mathrm{~K} \mathrm{~m}^{-1}$ and a slope of -1.0 [24]. The slope of the line through data set $(\mathrm{h})$, for silicon carbide particles in a succinonitrile melt, is -0.9 [1].

within a molecular diameter, the planar interface approximation would suggest a critical velocity of $v_{\mathrm{c}} \approx 10^{2} \mu \mathrm{s}^{-1}$ which seems unrealistically rapid.

When the leading edge of the particle sinks below the $T_{\mathrm{m}}$ isotherm and $h$ is less than unity, a different approximation for $\delta$ becomes appropriate. To be precise, when $\varepsilon \delta_{0}$ is much less than $1-h$, Eq. (11) reduces to

$\delta^{-3} \approx \varepsilon^{-1}(\cos \theta-h)$.

This is equivalent to approximating the temperature at the solid-liquid interface with the temperature on the surface of the particle. Since the integrals in Eq. (13) receive their largest contributions from small values of $\theta$, the exact value taken for $\theta_{\mathrm{c}}$ is not critical. As long as $h \ll 1-\varepsilon \delta_{0}$ we can write $\theta_{\mathrm{c}} \approx \cos ^{-1} h$ and evaluate the integrals in Eq. (13) to give the approximate dimensionless particle velocity as

$$
u \approx \varepsilon \frac{8+4 h}{3-2 h-h^{2}}
$$

Eq. (17) can be rewritten in terms of the film thickness by substituting $h \approx 1-\varepsilon \delta_{0}^{-3}$ so that the leading order $u \approx 3 \delta_{0}^{3}$. The dot-dashed line on the left side of Fig. 2 shows this approximation for the particle velocity at small film thickness. In this regime the particle velocity decreases monotonically as the particle is engulfed and the film becomes thinner. The distinction between the temperature on the particle surface and the temperature at the phase-change interface must be made in order to calculate the critical solidification velocity.

When the particle is completely captured and $h=-1$, the dimensionless particle velocity from Eq. (17) is $u \approx \varepsilon$. The velocity scale $W$ is thus revealed to be $\varepsilon^{-1}$ times the velocity at which the particle migrates by thermal regelation immediately after it has become trapped within the ice. This indicates that the solidification velocity at which a particle is captured is much more rapid than the velocity with which the particle can migrate within the ice towards warmer temperatures. After the particle is engulfed within the ice and $h<-1$, the formula given in Eq. (17) is no longer appropriate because the limit of integration $\theta_{\mathrm{c}}=\cos ^{-1} h$ used to evaluate the integrals in Eq. (13) becomes invalid. Further analysis using $\theta_{\mathrm{c}}=\pi$ reveals that the particle continues to travel up the temperature gradient at a regelation velocity of $U \approx-\varepsilon W / h$ after it is completely trapped within the ice and $h \leqslant-1$.

The dotted lines in Fig. 2 give the predictions of Chernov and colleagues [8] for $u$. The lower curve uses a small $\theta$ approximation for the film thickness, which is equivalent to approximating the interface shape as a paraboloid. The qualitative behaviour is similar to that of the leading order solution given by Eq. (14), but the magnitude of the critical solidification velocity $V_{\mathrm{c}}$ is underestimated by a factor of two, and the position of the maximum is displaced to a larger film thickness. The upper dotted line is derived using an interface shape that consists of a paraboloid that intersects at a small angle with 
the plane of the undeformed interface. This approximation yields predictions that are very close to those given by Eq. (14), which results from a more rigorous derivation.

Fig. 3 displays experimental data from a number of studies that show how the critical solidification velocity changes with the particle radius. Each symbol on the graph corresponds to a different particle type or temperature gradient. A best-fit line is projected through each of the data sets. The slopes of these lines reveal the observed dependence between the critical solidification velocity and the particle radius. The predicted inverse relationship between $V_{\mathrm{c}}$ and $R$ is consistent with the experimental data for most of these studies. The exceptions are the experiments with tungsten (data set $c$ ) [19] and one set of experiments with latex particles (data set $e$ ) [21] where the data is represented best by lines with slopes of -0.4 and -0.6 , respectively. However, each of the best-fit lines through the remaining seven data sets has a slope near the predicted value of negative one.

The thermomolecular pressure that repels the particle from the ice-liquid interface accounts for only one component of the total difference between the pressure in the ice phase $P_{\mathrm{s}}$ and the pressure in the liquid $P_{1}$. When the phase boundary is highly curved, the surface energy of the interface $\sigma_{\mathrm{sl}}$ can also be responsible for a significant contribution to the pressure difference $P_{\mathrm{s}}-P_{1}$. Including the effects of interface curvature, the modified form of Eq. (3) for the film thickness can be written as

$\frac{l^{v+1}}{d^{v}}+\Gamma \kappa=(R+d) \cos \theta-H$,

where $\kappa \equiv R K$ is the dimensionless interface curvature and the length scale $\Gamma \equiv\left(T_{\mathrm{m}} \sigma_{\mathrm{sl}}\right) /\left(\rho q_{\mathrm{m}} G R\right)$. The magnitude of the dimensionless interface curvature approaches unity when the particle nears the interface and the film thickness is comparable to the length scale $l$. Eq. (18) suggests that curvature effects are negligible when the length scale $l \gg \Gamma$ or equivalently when the particle radius $R \gg R_{\mathrm{c}}$ with the critical radius $R_{\mathrm{c}}$ given by

$$
R_{\mathrm{c}}=\frac{\sigma_{\mathrm{sl}}}{\rho q_{\mathrm{m}}}\left(\frac{T_{\mathrm{m}}}{\lambda G}\right)^{v /(v+1)},
$$

where $\sigma_{\mathrm{sl}} \approx 0.03 \mathrm{Jm}^{-2}$ [25]. When nonretarded van der Waals forces dominate the intermolecular interactions, $v=3$ and $R_{\mathrm{c}} \approx 10^{2} \mu \mathrm{m}$ under typical experimental conditions. Data set $g$ is the only data set displayed in Fig. 3 that pertains to particles larger than the critical radius $R_{\mathrm{c}}$ given by Eq. (19); hence Eq. (3) for the film thickness is not actually valid under the conditions present during most of the experiments summarised in Fig. 3. When the particle radius is much larger than the critical radius $R_{\mathrm{c}}$, the thermomolecular pressure $P_{\mathrm{T}}$ is much greater than the pressure due to interface curvature so that $P_{\mathrm{s}}-P_{1} \approx P_{\mathrm{T}}$ and Eq. (3) holds.

Experimental efforts aimed at determining the relationship between the temperature gradient and the critical velocity have been much more limited. Fig. 4 depicts the results of two such studies. The solid line through data set $a$ has a slope of 0.5 , while the dashed line through data set $b$ has a slope of 0.35 . If the point corresponding to the lowest temperature gradient and critical velocity in data set $b$ is omitted, the best-fit line through the remaining points has a slope of 0.2. Evidently, the predicted dependence of the critical velocity on $G^{1 / 4}$ is not well established. Once again the particles used in these two studies were smaller than the critical radius given by Eq. (19). In addition, it should be

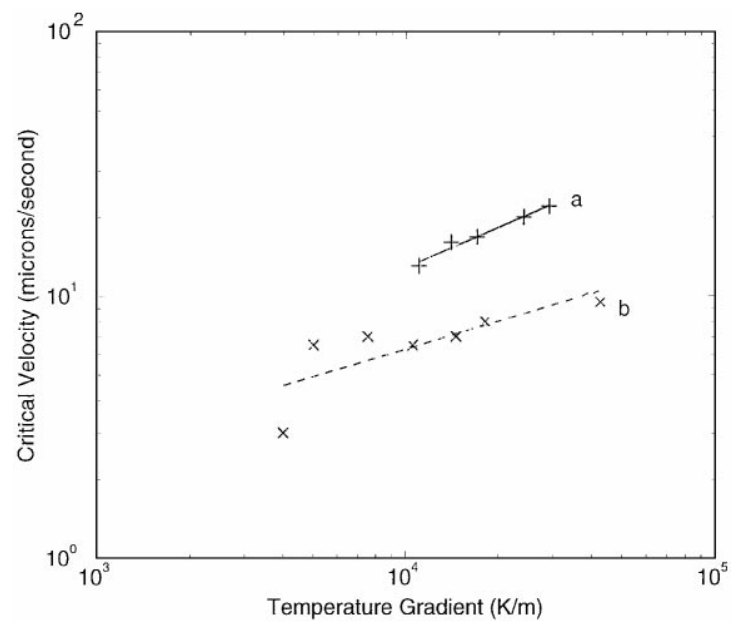

Fig. 4. The dependence of the critical velocity on the temperature gradient. Data set $a$ is for $5.7 \mu \mathrm{m}$ radius latex particles [20]; the best-fit solid line through the data has a slope of 0.5 . Data set $b$ is for $7 \mu \mathrm{m}$ radius latex particles [22]; the best-fit dashed line has a slope of 0.35 . 
noted that the temperature gradient is difficult to control and measure during an experiment. Indeed, the temperature gradient can change considerably over the course of a single experimental run. Further experimental efforts are required to demonstrate the precise dependence of $V_{\mathrm{c}}$ on $G$.

\section{Particle displacement}

If the solidification velocity exceeds the critical velocity, the particle will eventually become trapped within the solid. The distance it is pushed is

$D(t)=\int_{0}^{t} U \mathrm{~d} t=\int_{d_{0}(t=0)}^{d_{0}(t)} U \frac{\mathrm{d} t}{\mathrm{~d} d_{0}} \mathrm{~d} d_{0}$,

where $d_{0}(t=0)$ is the initial film thickness at $\theta=0$ and $d_{0}(t)$ is the film thickness at time $t$. The change in film thickness with time is found from an implicit differentiation of Eq. (3). The dimensionless displacement $\Delta$, scaled in terms of the particle radius, becomes

$\Delta(t)=\frac{D}{R}=\varepsilon \int_{\delta_{0}(t=0)}^{\delta_{0}(t)}\left(1+\frac{3}{x^{4}}\right)\left(\frac{u}{u-v}\right) \mathrm{d} x$,

where $v$ is the dimensionless solidification velocity (i.e. $v \equiv V / W$ ). If the solidification velocity is close to the critical velocity, the denominator $u-v$ becomes small near $\delta_{0}=1$ and the particle displacement can be significant.

The derivation for the particle velocity is applicable when the film thickness immediately beneath the particle is much less than the particle radius. With this in mind, we choose an initial dimensionless film thickness that is much larger than unity, but much less than $\varepsilon^{-1}$. Initially, for large film thickness $u \approx \delta_{0}^{-1}$ and the particle displacement is roughly

$\Delta \approx \frac{\varepsilon}{v} \ln \frac{\delta_{0}(t=0)}{\delta_{0}(t)}$.

As the particle nears the interface its velocity rises rapidly and the displacement increases more quickly. When the film thickness is small and $u \approx 3 \delta_{0}^{3}$ the particle displacement depends logarithmically on $\delta_{0}$ once again.

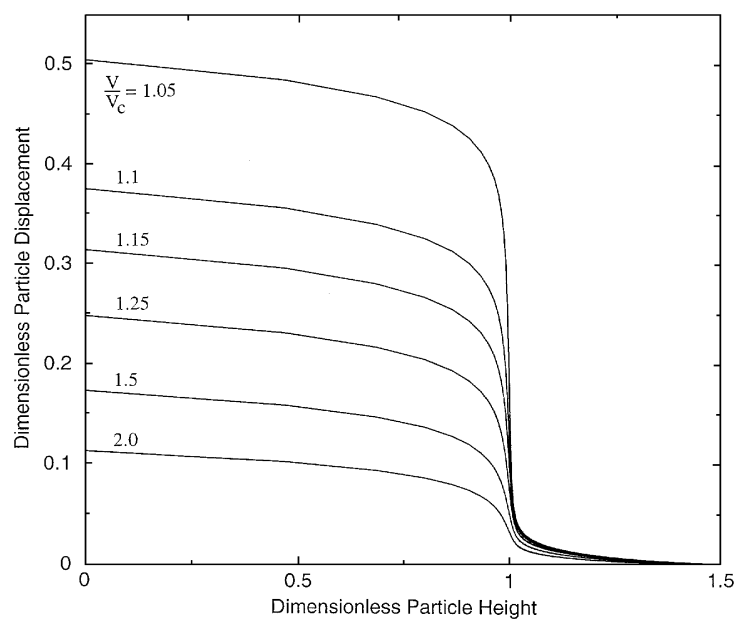

Fig. 5. Dimensionless particle displacement $\Delta$ as a function of the dimensionless particle height $h$, both scaled in terms of the particle radius. Eq. (21) was used for the calculations with each curve corresponding to a different ratio of the solidification velocity $v$ to the critical velocity $v_{\mathrm{c}}$ as noted on the graph. The particle displacement decreases rapidly as the solidification velocity becomes significantly larger than the critical velocity.

Fig. 5 shows a plot of the particle displacement as a function of the dimensionless height $h$ for different values of the solidification velocity $v$. Far from the interface the particle velocity is much less than the solidification velocity and the particle barely moves. Near $h=1$ the particle velocity reaches its maximum and there is a correspondingly large increase in $\Delta$. The peak in the particlevelocity film-thickness curve is extremely narrow, however, so that even for the slowest solidification velocity displayed, just 5\% greater than the critical velocity, the total displacement is only a fraction of the particle radius. This result is consistent with the observations of Azouni and colleagues [26] who measured particle displacements of up to a few particle diameters in a series of solidification experiments involving coated nylon spheres.

\section{Different interfacial interaction types}

The results presented thus far have been predicated on the assumption that nonretarded van der Waals forces control the film thickness. In many systems a more dominant role is played by 
other interfacial forces. However, the dependence of the film thickness on the interfacial undercooling can often be described by a power law of the form given in Eq. (1). For example, retarded van der Waals forces can be modelled by setting $v=4$, while for long range electrical interactions $v=2$ [13].

If we allow $v$ to take some value other than 3 , the length scale $l$ is conveniently generalised so that $l \equiv\left(\lambda^{v} T_{\mathrm{m}} / G\right)^{1 /(v+1)}$. The generic form of Eq. (3) for the film thickness as a function of position becomes

$\frac{l^{v+1}}{d^{v}}=(R+d) \cos \theta-H$.

We focus on the case in which the particle radius $R$ is much larger than the critical radius $R_{\mathrm{c}}$ from Eq. (19) so that the curvature of the phase boundary does not affect the film thickness. The thermomolecular pressure is directly proportional to the interfacial undercooling $T_{\mathrm{m}}-T_{\mathrm{i}}$ so that for arbitrary $v$ we can write

$P_{\mathrm{T}}=\rho q_{\mathrm{m}} \frac{T_{\mathrm{m}}-T_{\mathrm{i}}}{T_{\mathrm{m}}}=\rho q_{\mathrm{m}} \frac{\lambda^{v}}{d^{v}}$

Integrating $P_{\mathrm{T}}$ over the deformed interface in the same manner as demonstrated in Eq. (5) gives the thermomolecular force as

$F_{\mathrm{T}}=\left(2 \pi R^{2}\right) \rho q_{\mathrm{m}} \lambda^{v} \int_{0}^{\theta_{\mathrm{c}}} \frac{\sin \theta \cos \theta}{d^{v}} \mathrm{~d} \theta$.

Eq. (9) for the lubrication force is not affected by changing the interaction type. Balancing the forces on the particle using Eqs. (9) and (25) allows the particle velocity to be written as

$U=-\frac{\rho q_{\mathrm{m}} \lambda^{v}}{6 \mu R^{2}} \frac{\int_{0}^{\theta_{\mathrm{c}}} \sin \theta \cos \theta d^{-v} \mathrm{~d} \theta}{\int_{0}^{\theta_{\mathrm{c}}} \sin \theta \cos \theta\left(\int_{\theta_{\mathrm{c}}}^{\theta} \sin \phi d^{-3} \mathrm{~d} \phi\right) \mathrm{d} \theta}$.

The film thickness is naturally nondimensionalised using $d=\delta l$ as before. The change in the definition of $l$ brings about a slight modification to the velocity scale from Eq. (12) so that

$W \equiv \frac{\rho q_{\mathrm{m}} l^{3} G}{6 \mu T_{\mathrm{m}} R}=\frac{\rho q_{\mathrm{m}} \lambda^{3 v /(v+1)} G^{(v-2) /(v+1)}}{6 \mu T_{\mathrm{m}}^{(v-2) /(v+1)} R}$.
The velocity scale remains inversely proportional to the particle radius independently of the type of power-law interaction between the particle and the interface. The dependence of $W$ on the temperature gradient is, however, a function of the value of $v$. In fact, for electrical interactions that can be modelled by setting $v=2$ the velocity scale is independent of the temperature gradient. In this special case, the lubrication force and the thermomolecular force share the same dependence on the temperature gradient, whereas when $v$ is greater than two, the thermomolecular force increases more rapidly with $G$ and the particle velocity must rise to enable $F_{\mu}$ and $F_{\mathrm{T}}$ to balance. Using Eq. (27) for $W$, the dimensionless velocity becomes

$u=\frac{U}{W}=-\frac{\varepsilon \int_{0}^{\theta_{\mathrm{c}}} \sin \theta \cos \theta \delta^{-v} \mathrm{~d} \theta}{\int_{0}^{\theta_{\mathrm{c}}} \sin \theta \cos \theta\left(\int_{\theta_{\mathrm{c}}}^{\theta} \sin \phi \delta^{-3} \mathrm{~d} \phi\right) \mathrm{d} \theta}$

where $\varepsilon \equiv l / R$ as before. Once again the integrals in Eq. (28) can be transformed in order to integrate over the varying film thickness rather than the angular position. When $v \geqslant 2$, the leading-order behaviour is given by

$$
\begin{gathered}
u \approx\left(\frac{1}{2 \delta_{0}^{2 v}}+\frac{1}{(v-1) \delta_{0}^{v-1}}\right)\left(\frac{v^{2}}{(v+3)(2 v+3)} \frac{1}{\delta_{0}^{2 v+3}}\right. \\
\left.+\frac{v(v+5)}{2(v+3)(v+2)} \frac{1}{\delta_{0}^{v+2}}+\frac{1}{2 \delta_{0}}\right)^{-1}
\end{gathered}
$$

Approximations for $u$ at large and small values of $\delta_{0}$ can be readily derived using the same arguments as before.

Fig. 6 shows the dimensionless particle velocity as a function of the dimensionless particle height when $d$ is governed by Eq. (1) with $v=2,3$, and 4, corresponding to electrical interactions, nonretarded van der Waals interactions, and retarded van der Waals interactions, respectively. The solid line indicates that when $v=3$, the dimensionless critical velocity is roughly $u \approx 1$ as we have seen before (see Fig. 2). The dotted line, corresponding to $v=4$, has a peak at $u \approx 0.8$ when $h \approx 1$ and the base of the particle is level with the bulk melting temperature isotherm. At larger values of $v$ the interfacial interactions are dominated by an increasingly limited region immediately beneath the particle where the film thickness is smallest. The thermomolecular force decays more rapidly with 


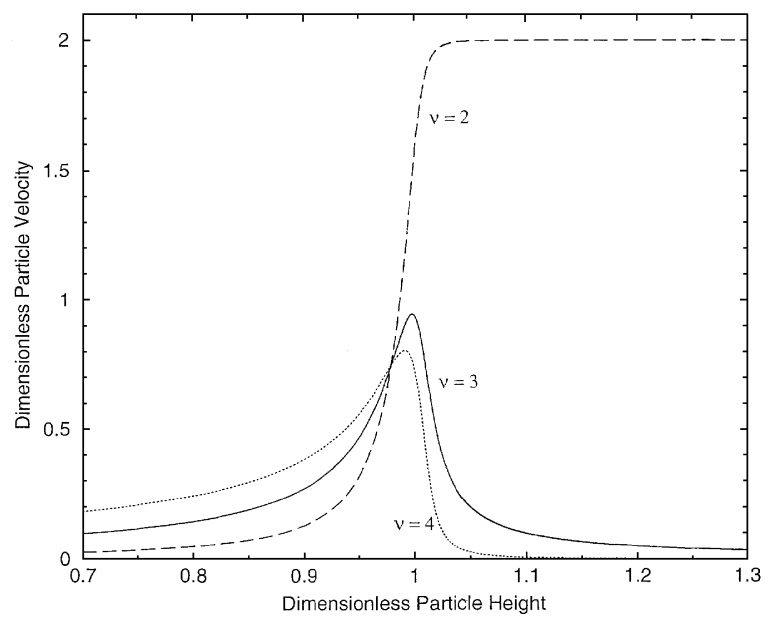

Fig. 6. Dimensionless particle velocity $u$ as a function of the dimensionless particle height $h$ for different types of interfacial interactions. Each line displays the predicted behaviour for a different interaction type and hence a different value of $v$. The solid line is for nonretarded van der Waals interactions where $v=3$. The dotted line is for retarded van der Waals interactions with $v=4$. The dashed line represents electrical interactions that are modelled using $v=2$.

height $h$ when $v$ is higher so the particle velocity must decrease in order to enable a reduction in the lubrication force. When $v=2$, as seen by the dashed line in Fig. 6, the present theory suggests that the particle velocity tends to a constant value of $u \approx 2$ once the dimensionless particle height is slightly greater than unity. The formulation presented here is based on the assumption that the forces on the particle are dominated by the region where the surface of the particle and the phase boundary are nearly parallel. This is no longer the case when $v$ is allowed to take values of 2 or less and hence the current theory becomes inadequate. Nevertheless, the essential behaviour of the system is still controlled by the balance between the thermomolecular force and the viscous force. The critical velocity should remain a function of the velocity scale $W$ given in Eq. (27).

\section{Buoyancy effects}

The preceding analysis has focused on predicting the outcome of the interactions between an insol- uble particle and an advancing solidification front under the simplest physically relevant conditions. The thermomolecular force that acts to repel the particle from the interface competes with the lubrication force that tends to promote particle encapsulation in order to determine the particle velocity as a function of key parameters such as the radius and the temperature gradient. When the density of the particle is significantly different from the density of the liquid, the buoyancy force can be important. The tendency for the particle to be captured within the growing solid phase can be either enhanced or reduced by gravitational effects, depending on the direction of the buoyancy force relative to the advancing solidification front. In this section we explore briefly how the particle buoyancy affects the behaviour of the system and the value of the critical velocity.

If $\Delta \rho$ is the density difference between the particle and the liquid, then the buoyancy force is

$F_{\mathrm{B}}=\frac{4}{3} \pi R^{3} g \Delta \rho$,

where $g$ is the acceleration of gravity. As the base of the particle nears the position of the bulk meltingtemperature isotherm, the particle velocity becomes comparable to the velocity scale $W$ from Eq. (27) and the film thickness beneath the base of the particle becomes comparable to the lengthscale $l$. The magnitude of the lubrication force from Eq. (9) becomes roughly

$F_{\mu} \approx 12 \pi \mu R^{2} \frac{W}{l}$.

We can compare the ratio of $F_{\mathrm{B}}$ from Eq. (30) to $F_{\mu}$ from Eq. (31) to find that the buoyancy force is negligible when the particle radius is much less than

$R_{\mathrm{B}} \equiv\left(\frac{9 \mu W R}{\lg \Delta \rho}\right)^{1 / 2}=\left(\frac{3 \rho q_{\mathrm{m}} \lambda^{3 / 2} G^{1 / 2}}{2 T_{\mathrm{m}}^{1 / 2} g \Delta \rho}\right)^{1 / 2}$,

where the second equality is appropriate when the velocity scale $W$ and the length scale $l$ are defined with $v=3$. When nonretarded van der Waals forces dominate and the density ratio $\Delta \rho / \rho$ is near unity, the particle radius at which buoyancy effects begin to become important is roughly $10 \mu \mathrm{m}$. The force balance is modified so that the leading-order expression from Eq. (29) for the dimensionless 
particle velocity becomes

$$
\begin{gathered}
u \approx\left(f_{\mathrm{B}}+\frac{\delta_{0}^{-2 v}}{2}+\frac{\delta_{0}^{1-v}}{v-1}\right)\left(\frac{v^{2} \delta_{0}^{-2 v-3}}{(v+3)(2 v+3)}\right. \\
\left.+\frac{v(v+5) \delta_{0}^{-v-2}}{2(v+3)(v+2)}+\frac{\delta_{0}^{-1}}{2}\right)^{-1},
\end{gathered}
$$

where the dimensionless buoyancy force $f_{\mathrm{B}} \equiv \pm R^{2} / R_{\mathrm{B}}^{2}$ is negative when gravity causes the initial particle motion to be towards the solidification front.

Fig. 7 shows the particle velocity from Eq. (33) as a function of the film thickness for several different values of the dimensionless buoyancy force $f_{\mathrm{B}}$ with the intermolecular interactions dominated by nonretarded van der Waals forces so that $v=3$. When the buoyancy of the particle is towards the solidification front, $f_{\mathrm{B}}$ is negative and the particle velocity is reduced from the value it would have if the effect of gravity were negligible. In this case the particle motion is towards the interface when the film thick-

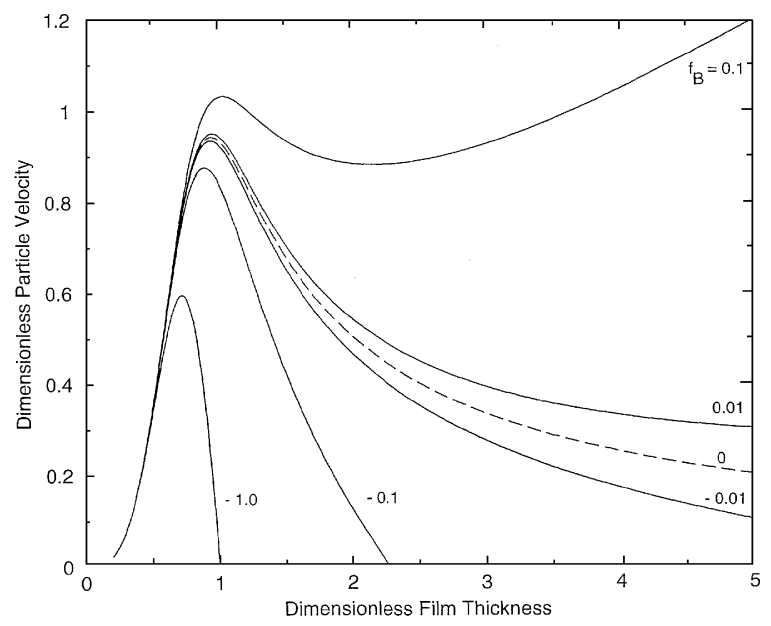

Fig. 7. Dimensionless particle velocity $u$ as a function of the dimensionless film thickness $\delta_{0}$ for large particles where the intermolecular interactions are dominated by nonretarded van der Waals forces. The dashed line is the velocity that is predicted when the particle radius is much larger than $R_{\mathrm{B}}$ and buoyancy effects are negligible. The solid lines correspond to the predictions of Eq. (33) for different values of the dimensionless buoyancy force $f_{\mathrm{B}}$ as noted on the graph. When $f_{\mathrm{B}}$ is negative, the effects of gravity tend to promote particle encapsulation and the critical velocity is reduced. When $f_{\mathrm{B}}$ is greater than zero the particle tends to migrate away from the interface under the influence of gravity and the particle velocity is more rapid. ness is large so that $u$ is negative at large $\delta_{0}$. At some finite film thickness, marked by the horizontal intercept of the curves in Fig. 7, the thermomolecular force becomes larger than the buoyancy force and the particle begins to move in the same direction as the solidification front. The particle velocity increases as the film thins further until $u$ either matches the solidification velocity or the critical velocity is reached and the particle is trapped within the solid phase. Both the magnitude of the critical velocity and the film thickness at which it occurs are reduced at negative $f_{\mathrm{B}}$.

When the density difference is such that $f_{\mathrm{B}}$ is positive, the effect of gravity is to pull the particle away from the interface. In the theory developed thus far, the particle velocity is predicted to be larger than the solidification velocity when the film thickness is large and $f_{\mathrm{B}}>0$. This simply reflects the fact that gravity acts to transport the particle away from the solidification front when the buoyancy force is positive. We have not taken into account the viscous drag that is associated with fluid flow around the bulk of the particle. When the film thickness is small, the drag is dominated by the lubrication force that accompanies fluid flow in the thin film that separates the particle from the phase boundary. At larger film thickness, the viscous drag around the bulk of the particle is more important. This bulk component of the drag, which can be approximated simply as Stokes' drag, balances the buoyancy force so that the terminal particle velocity

$$
U_{\mathrm{T}} \approx \frac{2 g R^{2} \Delta \rho}{9 \mu},
$$

is reached at large film thickness. If the terminal velocity is greater than the solidification velocity, the particle and the interface will separate and never interact. The dimensionless form of the terminal velocity can be written as

$u_{\mathrm{T}} \equiv \frac{U_{\mathrm{T}}}{W}=\frac{2 f_{\mathrm{B}}}{\varepsilon}$.

When $f_{\mathrm{B}}$ is of order $\varepsilon$, in addition to being comparable to the critical velocity, the terminal velocity can be less than the solidification velocity. If $u_{\mathrm{T}}$ is less than $v$, the solidification front will overtake the 
particle and the film thickness will be reduced. The thermomolecular force causes the particle velocity to increase as the dimensionless film thickness nears unity and $u$ either matches the solidification velocity or the critical velocity is reached. When $f_{\mathrm{B}}$ is positive, the critical velocity is more rapid than it would be without the effect of gravity, and it occurs at a slightly larger film thickness.

\section{Conclusions}

As a solidification front nears a particle, intermolecular forces between the particle, the solid, and the liquid can act to repel the particle from the phase boundary. This repulsion is opposed by viscous forces in the premelted film that forms between the particle and the deformed interface. By balancing these two forces we have shown that the velocity of the particle reaches a maximum when the leading edge of the particle is roughly level with the bulk melting-temperature isotherm. The solid-liquid interface deforms so that the thickness of the film separating the particle from the interface depends on the strength of the intermolecular interactions, the temperature gradient, and the melting temperature of the bulk solid. This interface deformation must be accounted for in order to calculate the maximum (critical) particle velocity. If the solidification velocity is greater than the critical velocity, the particle eventually becomes trapped within the growing solid region. When the dependence of the film thickness on the undercooling takes the form of a power law, the critical velocity is inversely proportional to the particle radius. The critical velocity is generally less sensitive to the temperature gradient, and the dependence is a function of the type of intermolecular forces that dominate the system behaviour. For example, if nonretarded van der Waals forces control the film thickness, the critical velocity depends on the temperature gradient raised to the one fourth power. The particle is pushed some distance ahead of the phase boundary even when the solidification velocity exceeds the critical velocity. This particle displacement is significant only when the solidification velocity is very close to the critical velocity. Particle buoyancy can affect the behaviour of larger particles by either raising or lowering the critical velocity depending on the direction of the buoyancy force relative to the motion of the solidification front.

\section{Acknowledgements}

This work has benefitted from numerous discussions with J.S. Wettlaufer and from the insightful comments of A.A. Chernov and an anonymous reviewer.

\section{References}

[1] D. Shangguan, S. Ahuje, D.M. Stefanescu, Metall. Trans. A 23A (1992) 669.

[2] V.L. Bronstein, Y.A. Itkin, G.S. Ishkov, J. Crystal Growth 52 (1981) 345.

[3] A.E. Corte, J. Geophys. Res. 67 (1962) 1085.

[4] D.R. Uhlmann, B. Chalmers, K.A. Jackson, J. Appl. Phys. 35 (1964) 2986

[5] G.F. Bolling, J. Cissé, J. Crystal Growth 10 (1971) 56.

[6] R.R. Gilpin, J. Colloid Interface Sci. 74 (1980) 44.

[7] R.R. Gilpin, J. Colloid Interface Sci. 68 (1979) 235.

[8] A.A. Chernov, D.E. Temkin, A.M. Mel'nikova, Sov. Phys. Crystallogr. 21 (1976) 369

[9] A.A. Chernov, D.E. Temkin, in: E. Kaldis, H.J. Scheel (Ed.), Current Topics in Materials Science, Vol. 2, 1976, Crystal Growth and Materials, North-Holland, Amsterdam, 1977, p. 3.

[10] J. Israelachvili, Intermolecular and Surface Forces, Academic Press, London, 1992.

[11] M.G. Worster, J.S. Wettlaufer, in: W. Shyy (Ed.), Fluid Dynamics at Interfaces, Cambridge University Press, Cambridge, 1999, in press.

[12] J.G. Dash, H. Fu, J.S. Wettlaufer, Rep. Prog. Phys. 58 (1995) 115

[13] J.S. Wettlaufer, M.G. Worster, L.A. Wilen, J.G. Dash, Phys. Rev. Lett. 76 (1996) 3602.

[14] J.S. Wettlaufer, Phys. Rev. Lett. 82 (1999) 2516.

[15] S. Sen, W.F. Kaukler, P. Curreri, D.M. Stefanescu, Metall. Trans. A 28A (1997) 2129.

[16] L.A. Wilen, J.S. Wettlaufer, M. Elbaum, M. Schick, Phys. Rev. B 52 (1995) 12426.

[17] M. Elbaum, M. Schick, Phys. Rev. Lett. 66 (1991) 1713.

[18] P. Casses, M.A. Azouni, Int. Commun. Heat Mass Transfer 22 (1995) 605.

[19] J. Cissé, G.F. Bolling, J. Crystal Growth 10 (1971) 67.

[20] C. Korber, G. Rau, M.D. Cosman, E.G. Carvalho, J. Crystal Growth 72 (1985) 649.

[21] G. Lipp, C. Korber, G. Rau, J. Crystal Growth 99 (1990) 206. 
[22] G. Lipp, C. Korber, J. Crystal Growth 130 (1993) 475.

[23] M.A. Azouni, W. Kalita, M. Yemmou, J. Crystal Growth 99 (1990) 201

[24] S. Sen, B.K. Dhindaw, D.M. Stefanescu, A. Catalina, P.A. Curreri, J. Crystal Growth 173 (1997) 574.
[25] D.W. Oxtoby, in: J.S. Wettlaufer, J.G. Dash, N. Untersteiner (Eds.), Ice Physics and the Natural Environment, Springer, New York, 1999, p. 23.

[26] M.A. Azouni, P. Casses, B. Sergiani, Colloids Surf. A 122 (1997) 199. 